\title{
Series approximations for Rayleigh distributions of arbitrary dimensions and covariance matrices
}

DOI:

10.1016/j.sigpro.2019.06.035

\section{Document Version}

Accepted author manuscript

Link to publication record in Manchester Research Explorer

\section{Citation for published version (APA):}

Wiegand, M., \& Nadarajah, S. (2019). Series approximations for Rayleigh distributions of arbitrary dimensions and covariance matrices. Signal Processing, 165, 20-29. https://doi.org/10.1016/j.sigpro.2019.06.035

\section{Published in:}

Signal Processing

\section{Citing this paper}

Please note that where the full-text provided on Manchester Research Explorer is the Author Accepted Manuscript or Proof version this may differ from the final Published version. If citing, it is advised that you check and use the publisher's definitive version.

\section{General rights}

Copyright and moral rights for the publications made accessible in the Research Explorer are retained by the authors and/or other copyright owners and it is a condition of accessing publications that users recognise and abide by the legal requirements associated with these rights.

\section{Takedown policy}

If you believe that this document breaches copyright please refer to the University of Manchester's Takedown Procedures [http://man.ac.uk/04Y6Bo] or contact uml.scholarlycommunications@manchester.ac.uk providing relevant details, so we can investigate your claim.

\section{OPEN ACCESS}




\title{
Series approximations for Rayleigh distributions of arbitrary dimensions and covariance matrices
}

\author{
Martin Wiegand, Saralees Nadarajah \\ School of Mathematics, University of Manchester, Manchester M13 9PL, UK \\ email: mbbsssn2@manchester.ac.uk
}

July 12, 2019

\begin{abstract}
The multivariate Rayleigh distribution is of crucial importance to many applied problems of engineering, such as in the analysis of multi-antenna wireless systems. Due to the lack of a generalised closed form of the distribution, the dependence on effective approximation methods for evaluation has created numerous numerical approaches with considerable restrictions in both dimensionality, as well as the structure of covariance matrices. In this paper we extend a previously introduced method [1] without either of these limitations. We then compare the performance of the new algorithms to recent integration methods of fixed dimension, presented by Beaulie and Zhang [2] and highlight the advantages of the new method.
\end{abstract}

Keywords: Correlation, Covariance, Multivariate Rayleigh distribution, Signal processing

\section{Introduction}

The Rayleigh distribution is essential to various applications in the field of signal processing, being the most fundamental model to describe signal fading in wireless systems. Common applications include the computation of outage probabilities, as evidenced in much of the literature dedicated to the subject [2] [3] [4], or the interference of elementary waves [5]. One of the most frequent utilisation of multivariate Rayleigh distributions (along with Rician or Nagakami distributions) lies in the modelling and analysis of multi-antenna wireless communications systems, where the dimension of the distribution corresponds to the number of channels or antennae in the system [6]. A Rayleigh approximation without dimensionality constraints therefore allows for systems of arbitrary size.

So far no closed form density function for Rayleigh distributed random variables has been put forth, apart from the univariate case. Therefore fast and reliable numerical approximations are necessary to evaluate various Rayleigh-based models. Over the years many such approximations have been proposed, utilising series representations, integration methods or a combination of both. Current numerical evaluations are defined for a fixed number of dimensions, requiring new formulations for every dimension, while others may simply not be extended to more general cases. These approaches have been used with varying success and occasionally poor computational performance. Some of the most recent advances in approximation methods have been addressed or developed by Le [7] [8] [9] [10], or Beaulieu and Hemachandra [6] among others. However, while some of these have proposed approximations that generalise the number of channels in the observed systems, correlation structures remain restrictive by postulating equal correlations [7] [8] [9] or other specific structures between channels [6]. In an earlier work we introduced a 
generalised series representation, which can be used for distributions of arbitrary dimensions [1]. However, based on the original method, we introduced a similar restriction on the structure of the covariance matrix, requiring identical values on the minor diagonals. We denote the $k$ th minor diagonal as $a_{j, i}$ with $|j-i|=k$. In this paper we extend [1] by introducing arbitrary covariance matrices to the base approach.

This paper is structured as follows: After the introduction where we reviewed previous methods of approximation and their applications, we move on to Section 2 discussing the generalisation of [1] to arbitrary covariance matrices. In Section 3, we investigate the performance of our newly proposed series approximation and compare the results to one of the most recently proposed integration approximations [2]. To validate our claims we test all methods for a constructed and an arbitrary covariance matrix of both low and high correlations, all in a three and a four dimensional space. We investigate the convergence speed of the series expansion against the accompanying increase in computational effort. Section 4 offers insight into possible applications of the series representation by computing outage probabilities of multichannel systems. Lastly we conclude this paper by summarising our new findings and propose further research projects.

\section{Approximation method}

Virtually every approximation method exploits the Rayleigh distribution's relation to the length $\left(L^{2}\right.$ norm) of a normally distributed, multivariate random vector. This approach has been used for decades to derive fixed dimension densities [11] [12, by setting up an initial expression from which the Rayleigh distribution may be derived as a marginal. We construct an $n$-dimensional representation of the Rayleigh distribution, by introducing $2 n$ zero-mean Gaussian random variables $X=X_{1}, \ldots, X_{n}$ and $Y=Y_{1}, \ldots, Y_{n}$ with their respective variances $\sigma_{1}^{2}, \ldots, \sigma_{n}^{2}$. The variables $X_{i}$ and $X_{j}$ as well as $Y_{i}$ and $Y_{j}$ are correlated by $\rho_{i, j}$ for $i<j$, whereas the vectors $X$ and $Y$ remain uncorrelated to each other. In this setup, the random variable $X$ denotes the in-phase and $Y$ the quadrature part of a given signal. We hence start out with the joint Gaussian distribution in 1 for a given sample $X=\left(x_{1}, \ldots, x_{n}\right)$ and $Y=\left(y_{1}, \ldots, y_{n}\right)$ :

$$
f\left(x_{1}, \ldots, x_{n}, y_{1}, \ldots, y_{n}\right)=\frac{1}{(2 \pi)^{n}|\Sigma|} \exp \left[-\frac{(x, y)^{T} \Sigma^{-1}(x, y)}{2}\right] .
$$

We denote the combined covariance matrix $(X, Y)$ by $\Sigma$ :

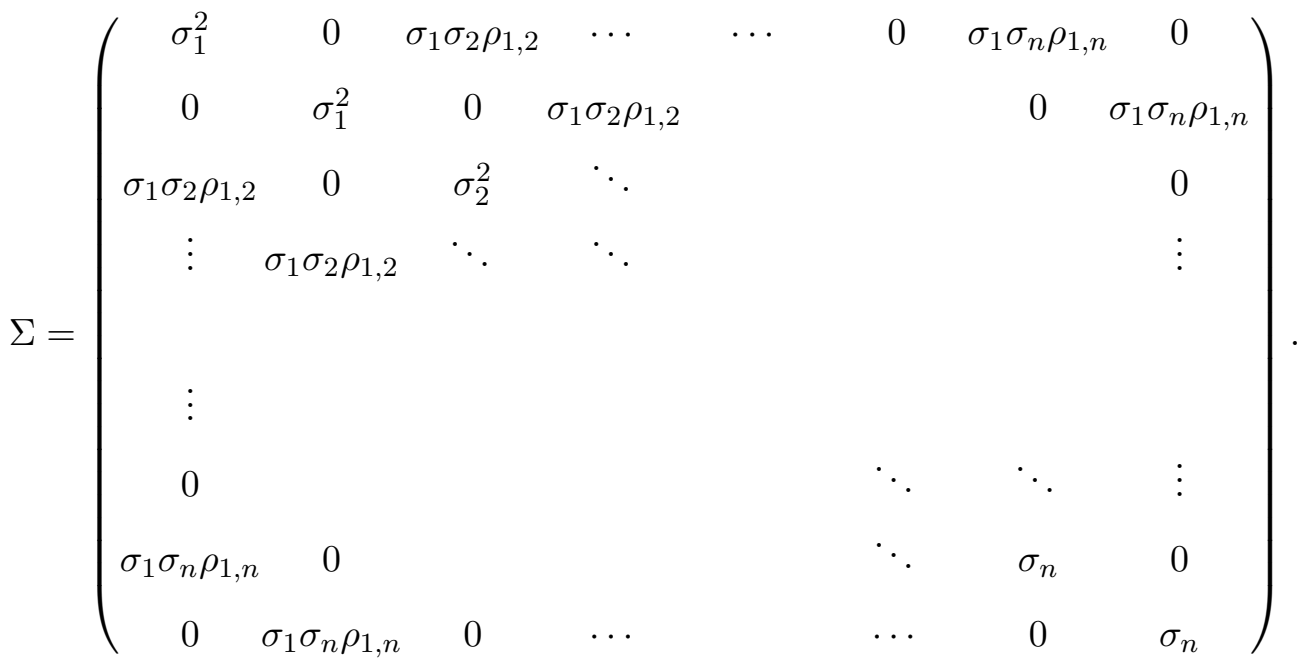


This stands in contrast to the previous approaches [1] and [3, where the correlations were limited to fixed values on the main and minor diagonals, such that $\rho_{i, j}=\rho_{i+k, j+k}$ remains the same for all $k \leq n-\max \{i, j\}$ (see [3] eq. 2).

Analogously to the approach of [1] we convert the cartesian coordinates into polar coordinates as $X_{i}=r_{i} \cos \theta_{i}$ and $Y_{i}=r_{i} \sin \theta_{i}$. The determinant of the Jacobian matrix of the transformation, $|J|=$ $r_{1} \cdots r_{n}$, appears as a factor in the density, and will be incorporated into the scalar factor later on. More importantly we use the identity $K^{-1}=1 /|\Sigma| C^{T}$, where $C \in \mathbb{R}^{2 n}$ is the cofactor matrix corresponding to $\Sigma$. The cofactor matrix retains the same sparse shape of the original matrix, as we can see in 3 .

$$
C=\left(\begin{array}{cccccccc}
c_{1,1} & 0 & c_{1,2} & 0 & \cdots & \cdots & c_{n} & 0 \\
0 & c_{1,1} & 0 & & & & & c_{n} \\
c_{1,2} & 0 & c_{2,2} & \ddots & & & & \vdots \\
0 & & \ddots & \ddots & & & & \\
\vdots & & & & & & & \\
& & & & & \ddots & \ddots & \vdots \\
\vdots & & & & & \ddots & c_{n, n} & 0 \\
c_{1, n} & & & & & \cdots & 0 & c_{n, n} \\
0 & c_{1, n} & \cdots & & & & & \\
& & & & & & & \\
& & & & & & & \\
& & & & & & &
\end{array}\right.
$$

The familiar formulation of 5 is obtained by performing the matrix-vector multiplication and the trigonometric identities $\sin (x)^{2}+\cos (x)^{2}=1$ and $\cos (a-b)=\cos (a) \cos (b)+\sin (a) \sin (b)$. In order to further clarify and streamline the notation, we denote the exponential coefficient in the product as $a_{i+j-2}=r_{i} r_{j} c_{i, j}$ with the index $t=1, \ldots, n(n-1) / 2=p$. Accordingly, we relabel the angle components $\bar{x}_{i+j-2}=\theta_{j}-\theta_{i}$ in the same way:

$$
\begin{aligned}
f\left(r_{1}, \ldots, r_{n}, \theta_{1}, \ldots, \theta_{n}\right) & =\underbrace{\frac{\prod_{i=1}^{n} r_{i}}{(2 \pi)^{n}|K|^{1 / 2}} \exp \left(-\frac{1}{2|K|} \sum_{i=1}^{n} r_{i}^{2} c_{i, i}\right)}_{=\gamma(r, c)} \prod_{i<j}^{n} \exp \left[-\frac{1}{|K|} c_{i, j} r_{i} r_{j} \cos \left(\theta_{j}-\theta_{i}\right)\right] \\
& \stackrel{(*)}{=} \gamma(r, c) \prod_{t=1}^{n(n-1) / 2} \exp \left[-\frac{1}{|K|} a_{t} \cos \left(\bar{x}_{t}\right)\right] .
\end{aligned}
$$

This constitutes in essence a conflation of multiple indices of different factor combinations, into a single variable with a single progressive index. In $(*)$ we substitute $x_{l-1}-x_{k-1}=\theta_{k}-\theta_{l}$, with $x_{0}=0$, as has been done in both [1] and [3. We can then proceed with 5 to derive the final density function by computing the marginal density by means of integration over the domain $[0,2 \pi]$ in 6 to derive the final 
result in 8 after some arithmetic and rearranging (for details see [1]):

$$
\begin{aligned}
f\left(r_{1}, \ldots, r_{n}\right) & =\int_{0}^{2 \pi} \cdots \int_{0}^{2 \pi} f\left(r_{1}, \ldots, r_{n}, \theta_{1}, \ldots, \theta_{n}\right) d \theta_{1} \ldots d_{n} \\
& =\gamma(r, c) \int_{0}^{2 \pi} \cdots \int_{0}^{2 \pi} \prod_{t=1}^{n(n-1) / 2} \exp \left[-\frac{1}{|K|} a_{t} \cos \left(\bar{x}_{t}\right)\right] d \theta_{1} \ldots d_{n} \\
& =\pi^{n} \gamma_{r, c} \sum_{j_{1}=0}^{\infty} \cdots \sum_{j_{p}=0}^{j_{p-1}} b_{t, j_{t}^{*}} \sum_{\rho \in\{-1,1\}^{p}} \prod_{w}^{n} \delta_{\left\{\Sigma_{x_{w}}=0\right\}} .
\end{aligned}
$$

The final formula of 8 makes use of the previous variables defined in 9 below:

$$
\begin{aligned}
& \Sigma_{x_{w}}=A_{w, 1}+\sum_{l=1}^{n-w} A_{l, w}+\sum_{l=1}^{w-1} A_{w-l, 1+l}
\end{aligned}
$$

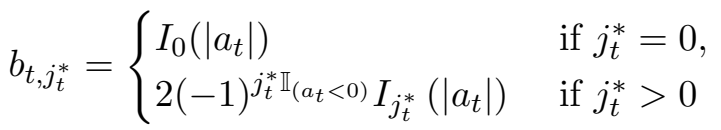

$$
\begin{aligned}
& j_{t}^{*}=j_{p-t+1}-j_{p-t+2} \\
& \alpha_{t}=j_{t}^{*} \rho_{t}
\end{aligned}
$$

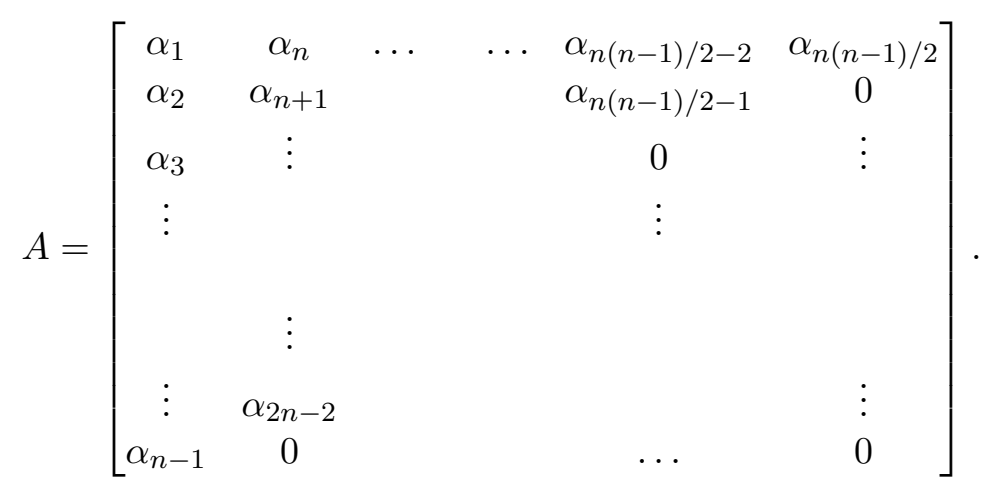

The relaxation of the restrictions on the covariance matrix gives us a much larger scope in potential application areas, and the basis for a truly universal formulation of the Rayleigh distribution, without dimensional limitations.

We have now assumed arbitrary cofactor matrix values $c_{i, j}$, and therefore arbitrary covariance matrix values $\Sigma_{i, i j}$. Therefore we may directly compare the newly derived series approximation to recent fixeddimension approaches without restrictions on correlation or covariance. The next section will assess the performance of both the series expansion and the integration-based method of Beaulieu and Zhang [2].

\section{Comparison}

The method developed by Beaulieu and Zhang [2] relies on the computation of the multivariate Rayleigh distribution by means of integrals necessary in the transition from joint Gaussian to marginal distributions ([1], 3]). The principal idea in this proposition is to determine two integrals analytically, leaving a formulation which consists of $n-2$ integrals, where $n$ is the dimension of the Rayleigh distribution. The 
remaining integrals are then to be evaluated by numerical means. Beaulieu and Zhang [2] provide explicit formulations for the three and four-dimensional Rayleigh densities. We have given the functions in 10 below:

$$
f\left(r_{1}, r_{2}, r_{3}\right)=\prod_{i=1}^{3} \frac{r_{i}}{\sigma_{i}^{2}} \exp \left\{-\frac{1}{2|\Omega|}\left[\frac{r_{i}^{2}\left(1-\rho_{j k}^{2}\right)}{\sigma_{i}^{2}}\right]\right\} \int_{0}^{\pi} \frac{\exp L_{2} \cos t_{2}}{\pi|\Omega|} I_{0}\left(\sqrt{L_{1}^{2}+L_{3}^{2}+2 L_{1} L_{3} \cos t_{2}}\right) d t_{2},
$$

where $i \neq j \neq k, \sigma^{2}$ denotes the variance of each Gaussian random variable, while $\rho_{l, m}$ denotes their respective correlation. The coefficients $L_{1}, L_{2}$ and $L_{3}$ are defined in Beaulieu and Zhang [2] as follows:

$$
L_{1}=\frac{r_{2} r_{3}\left(\rho_{2,3}\right)-\rho_{12} \rho_{13}}{\sigma_{2} \sigma_{3}|\Omega|}, \quad L_{2}=\frac{r_{1} r_{3}\left(\rho_{1,3}\right)-\rho_{12} \rho_{23}}{\sigma_{1} \sigma_{3}|\Omega|}, \quad L_{3}=\frac{r_{1} r_{2}\left(\rho_{1,2}\right)-\rho_{13} \rho_{23}}{\sigma_{1} \sigma_{2}|\Omega|} .
$$

To evaluate this formulation in practice, we will have to rely on numerical integration methods which determine the value of the remaining integrals. We select methods in Table 1 to provide a sufficiently

\begin{tabular}{|c|c|c|c|c|c|}
\hline & Name & Туре & Function & $\mathbf{R}$ package & Note \\
\hline \multirow{5}{*}{ 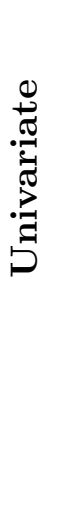 } & Kronrod & Quadrature & integral & pracma & \\
\hline & Simpson & Quadrature & integral & pracma & \\
\hline & TOMS614 & $\begin{array}{l}\text { Quadrature/ } \\
\text { Adaptive }\end{array}$ & int & rmutil & $\begin{array}{l}\text { Taken from the } \\
\text { ACM algorithm } \\
\text { collection }\end{array}$ \\
\hline & Romberg & Extrapolation & int & rmutil & \\
\hline & Mixed & Quadrature & integrate & stats & $\begin{array}{l}\mathrm{R} \text { interface for } \\
\mathrm{C}++ \text { code }\end{array}$ \\
\hline \multirow{3}{*}{ 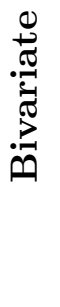 } & Romberg & Extrapolation & int2 & rmutil & \\
\hline & Quadrature & Cubature & quad2d & pracma & $\begin{array}{l}\mathrm{R} \text { wrapper for } \\
\text { MatLab code }\end{array}$ \\
\hline & Cubature & Cubature & pcubature & cubature & $\begin{array}{l}\mathrm{R} \text { wrapper for } \mathrm{C} \\
\text { code }\end{array}$ \\
\hline
\end{tabular}
varied picture of performances.

Table 1: An overview of the different numerical integration methods and their implementations.

Since there is no closed form of the multivariate Rayleigh distribution available to compare the approximations against, we need to select a boundary case for exact results. In order to do so, we chose the covariance matrix to simplify the integrand in 6 adequately to derive a closed form. In particular we need all entries of the cofactor matrix other than the main and the first minor diagonals to disappear. We may write this condition as $c_{i, j}=0$ for all $|i-j|>1$ (referred to as $\left({ }^{*}\right)$ ). The series expansion then 
collapses to the simpler case denoted in 15 .

$$
\begin{aligned}
f\left(r_{1}, \ldots, r_{n}\right) & =\gamma_{n, \Sigma, r} \int_{[0,2 \pi]^{n}} \prod_{t=1}^{p} \exp \left[a_{t} \cos \left(\overline{x_{t}}\right)\right] d x_{1} \cdots d x_{n} \\
& \stackrel{(* *)}{=} \gamma_{n, \Sigma, r} \int_{[0,2 \pi]^{n}} \exp \left[a_{1} \cos \left(x_{1}-x_{2}\right)+a_{n} \cos \left(x_{2}-x_{3}\right)+a_{2 n-3} \cos \left(x_{3}-x_{4}\right)+\cdots+a_{p} \cos \left(x_{n-1}-x_{n}\right)\right] d \\
& =\gamma_{n, \Sigma, r}(2 \pi)^{n} I_{0}\left(a_{1}\right) I_{0}\left(a_{n}\right)\left(a_{2 n-3}\right) \cdots I_{0}\left(a_{p}\right) \\
& =\frac{\prod_{i=1}^{n} r_{i}}{|\Sigma|^{1 / 2}} \exp \left(-\frac{1}{2|\Sigma|} \sum_{i=1}^{n} r_{i}^{2} c_{i, i}\right) \prod_{l=1}^{n-1} I_{0}\left(-\frac{1}{|\Sigma|} r_{l} r_{l+1} c_{l, l+1}\right)
\end{aligned}
$$

We note that the result in 15 constitutes a boundary case where approximation and true solution coincide, due to the zero-value sum terms of the approximation that disappear. This of course is because the random variable pairs $\left\{R_{i}, R_{j}\right\}$ for $k \leq|i-j|$, whose missing cofactors simplify the distribution significantly. The coefficient $\gamma$ is dependent on solely the covariance matrix and radius parameters and has been defined as follows:

$$
\gamma_{n, \Sigma, r}=\frac{\prod_{i=1}^{n} r_{i}}{(2 \pi)^{n}|\Sigma|^{1 / 2}} \exp \left(-\frac{1}{2|\Sigma|} \sum_{i=1}^{n} r_{i}^{2} c_{i, i}\right) .
$$

We have therefore produced a closed form solution for specific correlation and variance values. The formulation retrieved in 15 will be used as the true value, to which we will compare the approximations of the integral and series representation approaches. In order to quantify the accuracy of the proposed methods we will settle on a number of familiar error measures. Across an equidistant multidimensional grid, we will evaluate the true solution, the integration-based method for multiple numerical integration approaches, as well as the series expansion with different numbers of sum terms. We have computed the average absolute error (AAE), average relative absolute error (ARAE - in percentages), aggregated absolute error (AGAE) and the maximum absolute error (MAE):

$$
\begin{aligned}
A A E & =\frac{1}{N} \sum_{i}^{N}\left|\widehat{f}\left(x_{i}\right)-f\left(x_{i}\right)\right|, \\
A R A E & =\frac{100}{N} \sum_{i}^{N}\left|\frac{\widehat{f}\left(x_{i}\right)-f\left(x_{i}\right)}{f\left(x_{i}\right)}\right|, \\
A G A E & =\sum_{i}^{N}\left|\widehat{f}\left(x_{i}\right)-f\left(x_{i}\right)\right| \\
M A E & =\max _{i}\left|\widehat{f}\left(x_{i}\right)-f\left(x_{i}\right)\right| .
\end{aligned}
$$

The evaluation points are equidistantly distributed across an area $[0, b]^{n}$. The upper bound $b$ is chosen such that the hypercube encompasses the area of interest (until $f(b) \approx 0$ ). In addition to the accuracy we are concerned with the evaluation and setup speed of both procedures. With setup speed we refer to the computation time needed for the algorithm to determine the non-zero sum terms of the index value combinations $j_{1}, \ldots, j_{n}$. Whether or not an index value combination leads to a non-zero sum term is independent of the covariances, and can therefore be computed once and stored for other computations 
of the same dimension. To make the algorithm more transparent, we have decided to include the onetime computational effort for the algorithm setup regardless. We have hence recorded both times for all approaches, to give us an impression of the computational effort involved.

\subsection{Three-dimensional case}

In a first step we will investigate a constructed three-dimensional example. We have chosen the variances as $\sigma_{1}^{2}=1.5, \sigma_{2}^{2}=2, \sigma_{3}^{2}=1.5$ and the correlations as $\rho_{1,2}=-1 / \sqrt{3}, \rho_{2,3}=-1 / \sqrt{3}, \rho_{1,3}=1 / 3$. The corresponding cofactor matrix is depicted in 21 .

$$
C=\left(\begin{array}{llllll}
4 & 0 & 2 & 0 & 0 & 0 \\
0 & 4 & 0 & 2 & 0 & 0 \\
2 & 0 & 4 & 0 & 2 & 0 \\
0 & 2 & 0 & 4 & 0 & 2 \\
0 & 0 & 2 & 0 & 4 & 0 \\
0 & 0 & 0 & 2 & 0 & 4
\end{array}\right)
$$

providing us the cofactor matrix structure necessary to gain a closed form boundary case of the Rayleigh density. We proceed to analyse how both approximations fared in the simulation. Table 2 lists the previously discussed metrics which characterise the performances of both methods. The upper part of the table is focused on the integration-based approaches performed by different numerical methods. The second half of the table depicts the error measures for the different numbers of sum terms of the series expansion. The grid has been created to span the cube $[0,3]^{3}$ and was evaluated at one million nodes $(f=100$ nodes in each dimension).

All computations were carried out on a personal computer, equipped with 4 Intel(R) Core(TM) i7$6700 \mathrm{CPU} @ 3.40 \mathrm{GHz}$ cores, which are divided into 8 Logical Processors and with 16GB available physical memory. While both setup and evaluation of our algorithm can be easily parallelised, we ran the computations on only one processor for better comparability. 


\begin{tabular}{|c|c|c|c|c|c|c|c|}
\hline $\begin{array}{l}f= \\
100\end{array}$ & TYPE & $\mathrm{AAE}$ & ARAE & AGAE & MAE & EVAL (min) & SETUP (s) \\
\hline \multirow{5}{*}{$\begin{array}{l}\bar{\varpi} \\
\stackrel{\sigma}{0} \\
\stackrel{0}{0} \\
\stackrel{\Xi}{ \pm}\end{array}$} & Kronrod & $2.21829 \mathrm{E}-11$ & 7.61392E-08 & $2.21829 \mathrm{E}-05$ & $1.27465 \mathrm{E}-10$ & 13.7299500 & 0.00000000 \\
\hline & TOMS614 & $2.40850 \mathrm{E}-08$ & 7.73793E-05 & $2.40850 \mathrm{E}-02$ & $1.67967 \mathrm{E}-07$ & 20.1209166 & 0.00000000 \\
\hline & Simpson & $2.21972 \mathrm{E}-11$ & $7.62012 \mathrm{E}-08$ & $2.21972 \mathrm{E}-05$ & $1.27465 \mathrm{E}-10$ & 49.8370500 & 0.00000000 \\
\hline & Mixed & $2.21829 \mathrm{E}-11$ & $7.61392 \mathrm{E}-08$ & $2.21829 \mathrm{E}-05$ & $1.27465 \mathrm{E}-10$ & 2.47048333 & 0.00000000 \\
\hline & Romberg & $2.26737 \mathrm{E}-11$ & 7.79200E-08 & $2.26737 \mathrm{E}-05$ & $1.27543 \mathrm{E}-10$ & 24.4264333 & 0.00000000 \\
\hline \multirow{8}{*}{$\begin{array}{l}\stackrel{\tilde{Q}}{\mathscr{E}} \\
\tilde{\omega}\end{array}$} & Closed & $4.61123 \mathrm{E}-18$ & $1.64066 \mathrm{E}-14$ & $4.61123 \mathrm{E}-12$ & $1.11022 \mathrm{E}-16$ & 0.24526666 & 0.00000000 \\
\hline & Series 3 & $3.83983 \mathrm{E}-18$ & $1.39695 \mathrm{E}-14$ & $3.83983 \mathrm{E}-12$ & $9.71445 \mathrm{E}-17$ & 2.38705000 & 0.24300003 \\
\hline & Series 6 & $3.83983 \mathrm{E}-18$ & $1.39695 \mathrm{E}-14$ & 3.83983E-12 & $9.71445 \mathrm{E}-17$ & 3.29476125 & 0.97500014 \\
\hline & Series 9 & $3.83983 \mathrm{E}-18$ & $1.39695 \mathrm{E}-14$ & $3.83983 \mathrm{E}-12$ & $9.71445 \mathrm{E}-17$ & 4.28667128 & 2.90371013 \\
\hline & Series 12 & $3.83983 \mathrm{E}-18$ & $1.39695 \mathrm{E}-14$ & $3.83983 \mathrm{E}-12$ & $9.71445 \mathrm{E}-17$ & 5.18513210 & 5.87441301 \\
\hline & Series 15 & $3.83983 \mathrm{E}-18$ & $1.39695 \mathrm{E}-14$ & $3.83983 \mathrm{E}-12$ & $9.71445 \mathrm{E}-17$ & 6.15813334 & 10.6629998 \\
\hline & Series 18 & $3.83983 \mathrm{E}-18$ & $1.39695 \mathrm{E}-14$ & $3.83983 \mathrm{E}-12$ & $9.71445 \mathrm{E}-17$ & 7.15973334 & 17.3380000 \\
\hline & Series 21 & $3.83983 \mathrm{E}-18$ & $1.39695 \mathrm{E}-14$ & $3.83983 \mathrm{E}-12$ & $9.71445 \mathrm{E}-17$ & 8.28696667 & 26.2049999 \\
\hline
\end{tabular}

Table 2: Performance table for three-dimensional approximations.

We can see that most of the integration algorithms perform similarly, with a rather low average error. All four error measures depict almost the same values within a very small margin for the integration approach. Only the TOMS614 algorithm seems to perform significantly worse, all error measures being a multiple of the values we see in the other four integration methods, by as much as a factor of 1000 . Compared to the series expansion however, the integration method performs worse by a wide margin, regardless of error measure or integration method $\left(\sim 2.2 \times 10^{-11}\right.$ vs $\left.3.8 \sim 10^{-18}\right)$. With only a single sum term used in the series expansion, which we have denoted as closed in the table, the expansion method already underbids the computed error measures by factors of $10^{7}, 10^{6}, 10^{7}$ and $10^{6}$ for the AAE, ARAE, AGAE and MAE, respectively. The subsequent series approaches of higher order show only little further improvement before stagnating at around $\sim 3.810^{-18}$. The differences in performance are at this point in the order of magnitude of the machine precision, and have therefore little to say about convergence or performance of the methods. In terms of the computation time, we suspected the series representation to be faster as the evaluation algorithm itself may appear more complex yet relies on basic operations, many of which were carried out before the series evaluation, regardless of parameter values. The highest order of series expansion completes the computation in $8.2 \mathrm{~s}$, whereas all the integration methods besides the mixed $\mathrm{C}$ method (which completes in $\sim 2.5$ ) are more time consuming. Our observations are largely matching our expectations, albeit with the exception of the "mixed" algorithm of the stats package. However, as we noted in Table 1 this numerical integration approach is merely an $\mathrm{R}$ frontend for $\mathrm{C}$ code, thus has to be somewhat disqualified for the sake of consistency, as the two programming languages have very different inherent evaluation speeds and computational efficiency.

We constructed 21 merely to verify the validity of our approximation in a testable boundary case. However, the example we created through the specific choice of covariance matrix may not be represen- 
tative for the problem as a whole, as the series expansion collapses to the true solution in this particular case. For arbitrary covariance matrices, no closed form does exist, which is why we are looking for an accurate approximation in the first place. In [1, we have derived the series representation as an exact expansion, which can be evaluated to arbitrary precision. To repeat our experiment in a more general setting, we have therefore chosen a series expansion of $n=30$ terms as a stand-in for the true solution, as the additional contributions for higher orders dropped below the machine precision (in the order of magnitude $\sim E-20$ ). We therefore define a second test example with parameters as follows: $\sigma_{1}^{2}=0.25$, $\sigma_{2}^{2}=0.1, \sigma^{2}=0.5$ and $\rho_{1,2}=0.3, \rho_{1,3}=-0.4, \rho_{2,3}=0.1$. This selection of variances and correlations does not pose a special case cofactor matrix, and can therefore be used to derive more meaningful results.

\begin{tabular}{|c|c|c|c|c|c|c|c|}
\hline $\begin{array}{l}f= \\
100\end{array}$ & TYPE & $\mathrm{AAE}$ & ARAE & AGAE & MAE & EVAL (min) & SETUP (s) \\
\hline \multirow{5}{*}{ 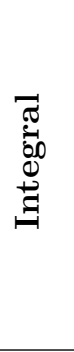 } & Kronrod & $2.00995 \mathrm{E}-11$ & $4.65180 \mathrm{E}-08$ & $2.00995 \mathrm{E}-05$ & $2.40802 \mathrm{E}-10$ & 10.0354333 & 0.00000000 \\
\hline & TOMS614 & $3.10563 \mathrm{E}-08$ & $6.94000 \mathrm{E}-05$ & $3.10563 \mathrm{E}-02$ & $5.94122 \mathrm{E}-07$ & 15.2123833 & 0.00000000 \\
\hline & Simpson & $2.00338 \mathrm{E}-11$ & $4.68059 \mathrm{E}-08$ & $2.00338 \mathrm{E}-05$ & $2.40802 \mathrm{E}-10$ & 46.1882500 & 0.00000000 \\
\hline & Mixed & $2.00995 \mathrm{E}-11$ & $4.65180 \mathrm{E}-08$ & $2.00995 \mathrm{E}-05$ & $2.40802 \mathrm{E}-10$ & 1.86520000 & 0.00000000 \\
\hline & Romberg & $3.55274 \mathrm{E}-11$ & $6.39262 \mathrm{E}-07$ & $3.55274 \mathrm{E}-05$ & $2.74488 \mathrm{E}-10$ & 19.9705666 & 0.00000000 \\
\hline \multirow{8}{*}{$\begin{array}{l}\frac{\mathscr{n}}{\tilde{D}} \\
\tilde{\tilde{D}}\end{array}$} & Series 1 & $1.18950 \mathrm{E}-03$ & $1.24626 \mathrm{E}+01$ & $1.18950 \mathrm{E}+03$ & $1.03932 \mathrm{E}-02$ & 0.18264999 & 0.00000000 \\
\hline & Series 3 & $1.64911 \mathrm{E}-05$ & $5.45667 \mathrm{E}-01$ & $1.64911 \mathrm{E}+01$ & $2.09788 \mathrm{E}-04$ & 1.78236667 & 0.18700004 \\
\hline & Series 6 & $1.82202 \mathrm{E}-07$ & $1.48143 \mathrm{E}-02$ & $1.82202 \mathrm{E}-01$ & $4.20331 \mathrm{E}-06$ & 2.47346667 & 0.79699993 \\
\hline & Series 9 & $1.59737 \mathrm{E}-09$ & $2.62467 \mathrm{E}-04$ & $1.59737 \mathrm{E}-03$ & $6.59571 \mathrm{E}-08$ & 3.16587333 & 2.03900003 \\
\hline & Series 12 & $1.11266 \mathrm{E}-11$ & $3.17883 \mathrm{E}-06$ & $1.11266 \mathrm{E}-05$ & $8.19534 \mathrm{E}-10$ & 3.87352927 & 4.53854609 \\
\hline & Series 15 & $6.17717 \mathrm{E}-14$ & $2.73157 \mathrm{E}-08$ & $6.17717 \mathrm{E}-08$ & $7.82583 \mathrm{E}-12$ & 4.62833467 & 7.81721807 \\
\hline & Series 18 & $2.74466 \mathrm{E}-16$ & $1.71696 \mathrm{E}-10$ & $2.74466 \mathrm{E}-10$ & $5.74052 \mathrm{E}-14$ & 5.37201666 & 13.5309999 \\
\hline & Series 21 & $9.80759 \mathrm{E}-19$ & 8.09972E-13 & $9.80759 \mathrm{E}-13$ & $3.32972 \mathrm{E}-16$ & 6.01856667 & 19.7210001 \\
\hline
\end{tabular}

Table 3: Performance table for three-dimensional approximations, for a random covariance matrix.

The various integration methods appear to perform roughly the same as in the previous test problem in terms of accuracy, with only minor differences, see Table 3 . The ARAE appears a little more volatile between methods than before, and the maximum absolute error across the domain has roughly doubled its value. The overall error values however remain largely the same, with the TOMS614 method once again performing noticeably weaker than the other integration processes, with each error measure about 1000 times higher compared to the other integration approaches. The different combination of variances and correlations in this setup does not immediately favour the series approach, and initially we get less accurate estimates for comparatively few sum terms, starting out at an average error of as high as $1.18 \times 10^{-3}$. At 12 sum terms we manage to match most integration methods in almost all performance categories (except for ARAE and MAE), and with 15 terms we have surpassed the benchmark method in every respect of accuracy.

The computation times for the integrals range from 10 to 45 minutes for one million evaluations. We 
believe that this many nodes do not only offer a greater accuracy in assessing the error measures, but also a more precise picture for the computational effort. Even at sum term numbers that enable the series expansion to perform more accurately than the integration approach, we have to invest less than half the evaluation time (6min maximum for 21 sum terms ). This is of course once again with the exception of the mixed C-code variant, which admittedly may have a practical use, but cannot be used to assess the computational complexity of the approximation method itself, as a series representation in C-code would undeniably be much faster than the R counterpart (which will be part of our further project development). The highest tested order of the series expansion takes little over 6 minutes to evaluate, with an accuracy that approaches machine precision at $\sim 9.810^{-19}$. Essentially we have shown that we can surpass the computational accuracy of the integration approach, while still preserving more efficient evaluation.

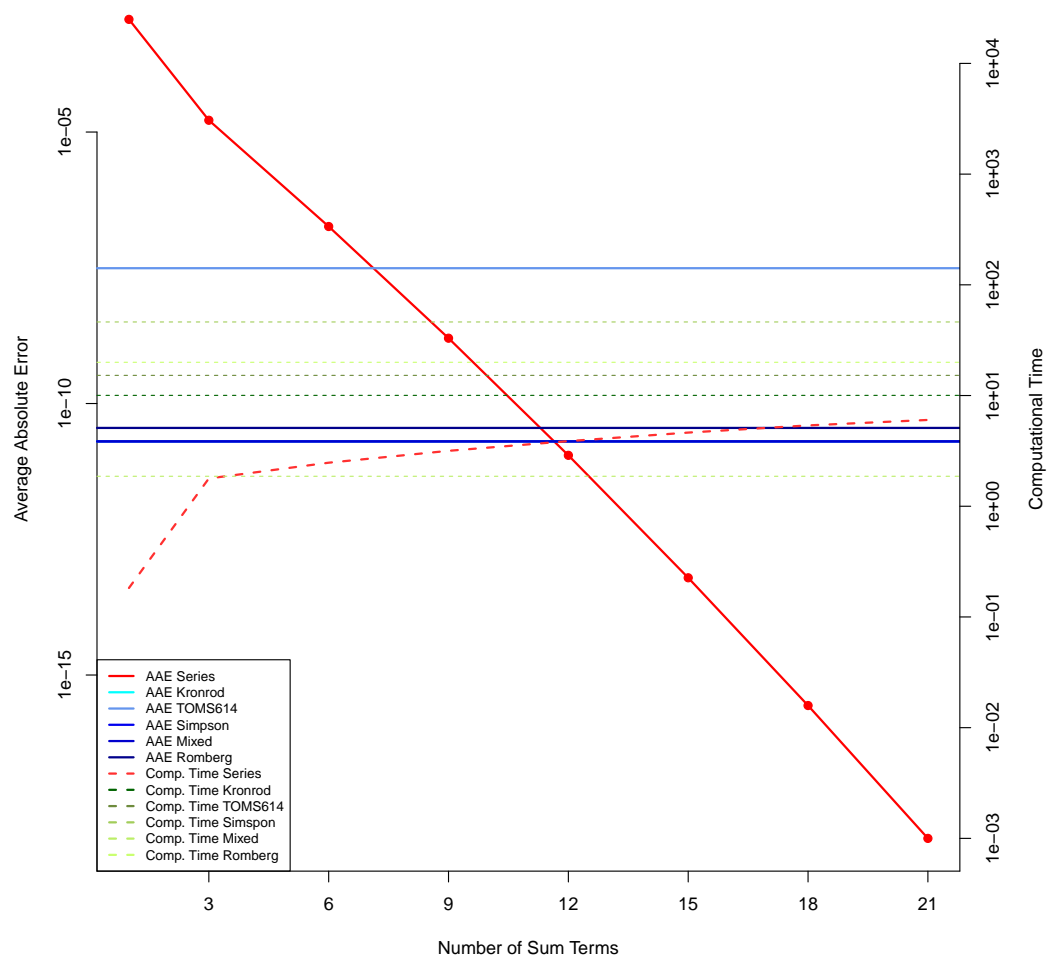

Figure 1: A graphical comparison between accuracy and runtimes of all tested three-dimensional approximation methods.

The last thing we need to address is the setup time. While the integration method can be evaluated at a given point directly, we need to compute a number of things before we carry on to determine the density at a given evaluation point. The setup time expectedly grows with increased order of the sum terms, but stays still within the bounds of less than 1 minute for the examples we have investigated. Also the growth in setup time appears to be diminishing as the number of sum terms increases, potentially suggesting a slower than linear growth relationship. Compared to the integration approach this may seem like a disadvantage for the computation of small sample sizes, as it increases the total computation time. However, the coefficients that are computed for each order of expansion are independent from the covariance matrix and any other parameters. Therefore we would have to do this computation for any given order of the series expansion only one time, regardless of covariance matrix. As a consequence we omit the setup time as an argument against the series expansion.

In Figure 1 we have visualised this performance disparity. The average absolute error decreases 
linearly in a logarithmic plot, suggesting an exponential relationship with the sum term order with a base of roughly 10. This shows the tremendous convergence speed of the series expansion, while the computational efficiency is only surpassed by the C-code implementation. However, as the main novelty of this series expansion is the lack of dimensional and covariance matrix restraints, we are eager to know how these behaviours react in higher dimensions, and if the disparity between the methods grows with a larger random vector.

Lastly we investigate one more set of correlations, to shed light on the impact of covariance matrices on the accuracy of the approximations. To this end we have chosen the variances as before $\sigma_{1}=0.5, \sigma_{2}=1$ and $\sigma_{3}=1.5$. The correlations in this experiment are set to $\rho_{1}=0.9, \rho_{2}=0.8$ and $\rho_{3}=0.7$, which cover the higher end of correlations. The results are depicted in Table 4 below.

\begin{tabular}{|c|c|c|c|c|c|c|c|}
\hline $\begin{array}{l}f= \\
100\end{array}$ & TYPE & AAE & ARAE & AGAE & MAE & EVAL (min) & SETUP (s) \\
\hline \multirow{5}{*}{ 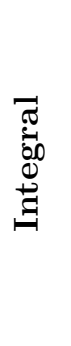 } & Kronrod & - & - & - & - & - & 0 \\
\hline & TOMS614 & $1.32279 \mathrm{E}-05$ & $9.62955 \mathrm{E}-02$ & $1.32279 \mathrm{E}+01$ & $6.09132 \mathrm{E}-04$ & 19.3434833 & 0 \\
\hline & Simpson & - & - & - & - & - & 0 \\
\hline & Mixed & $5.89940 \mathrm{E}-12$ & $1.85958 \mathrm{E}-08$ & $5.89940 \mathrm{E}-06$ & $3.13595 \mathrm{E}-10$ & 2.35811667 & 0 \\
\hline & Romberg & $4.45754 \mathrm{E}-09$ & $3.33035 \mathrm{E}-05$ & $4.45754 \mathrm{E}-03$ & $1.08388 \mathrm{E}-07$ & 18.7787000 & 0 \\
\hline \multirow{8}{*}{$\begin{array}{l}\stackrel{\infty}{\tilde{D}} \\
\stackrel{\infty}{\infty}\end{array}$} & Series 1 & $2.70535 \mathrm{E}-02$ & $1.16427 \mathrm{E}+02$ & $2.70535 \mathrm{E}+04$ & $5.70334 \mathrm{E}-01$ & 0.21988333 & 0 \\
\hline & Series 3 & $5.46290 \mathrm{E}-03$ & $2.88215 \mathrm{E}+01$ & $5.46290 \mathrm{E}+03$ & $2.26885 \mathrm{E}-01$ & 1.89770000 & 0.19199991 \\
\hline & Series 6 & 8.30757E-04 & $5.07632 \mathrm{E}+00$ & $8.30757 \mathrm{E}+02$ & $5.92135 \mathrm{E}-02$ & 2.61155000 & 0.80699992 \\
\hline & Series 9 & $9.88035 \mathrm{E}-05$ & $6.82823 \mathrm{E}-01$ & $9.88035 \mathrm{E}+01$ & $1.14786 \mathrm{E}-02$ & 3.38190000 & 2.05799985 \\
\hline & Series 12 & $9.44718 \mathrm{E}-06$ & $7.33837 \mathrm{E}-02$ & $9.44718 \mathrm{E}+00$ & $1.70428 \mathrm{E}-03$ & 4.06783333 & 4.47100019 \\
\hline & Series 15 & $7.41625 \mathrm{E}-07$ & $6.49326 \mathrm{E}-03$ & $7.41625 \mathrm{E}-01$ & $1.95733 \mathrm{E}-04$ & 4.87441667 & 7.71799994 \\
\hline & Series 18 & $4.86017 \mathrm{E}-08$ & $4.83162 \mathrm{E}-04$ & $4.86017 \mathrm{E}-02$ & $1.78832 \mathrm{E}-05$ & 5.53338333 & 12.7369999 \\
\hline & Series 21 & $2.69552 \mathrm{E}-09$ & $3.07124 \mathrm{E}-05$ & $2.69552 \mathrm{E}-03$ & $1.33273 \mathrm{E}-06$ & 6.48130000 & 18.4119999 \\
\hline
\end{tabular}

Table 4: Performance table for three-dimensional approximations, for a high correlation covariance matrix.

During the review of the results of the simulation, we quickly noticed that the Romberg and Simpson's integration methods did not show any error measures. This is due to the fact that after $24 \mathrm{~h}$ of runtime, the methods did not manage to procure the evaluations of the 1 million observed points. We therefore omit these methods from the analysis as impractical, or unsuited for high covariance ranges. The mixed method implemented in underlying $\mathrm{C}$ code is more flexible, and does not change much in terms of accuracy, and only marginally increased in computation time from $1.86 \mathrm{~min}$ to $2.35 \mathrm{~min}$.

For higher covariances the only directly comparable integration method is the Romberg method, which presents an accuracy of $\sim 4.45 \times 10^{-9}$ average absolute error and $\sim 3.3 \times 10^{-5}$ average relative error. With a computation time of $\sim 19 \mathrm{~min}$ the evaluation did not take significantly longer, but the accuracy has visibly decreased. The TOMS614 algorithm on the other hand can only reach an average absolute error as low as $1.32 \times 10^{-5}$, with an evaluation time of $19.34 \mathrm{~min}$. 
Similarly the series representation converges more slowly, and the highest displayed order of 21 sum terms reaches an AAE of $2.69 \times 10^{-9}$, which matches the Romberg methods accuracy. However, the evaluation time is around $\sim 6.5 \mathrm{~min}$, around 3 times lower than the Romberg integration methods needing for computation.

\subsection{Four-dimensional case}

Beaulieu and Zhang [2] offered a four-dimensional version of the previously stated approximation as well. We cite the four-dimensional extension directly from the source in 24 .

$$
\begin{aligned}
& f\left(r_{1}, r_{2}, r_{3}, r_{4}\right)= \\
& \quad \frac{1}{|\Omega|} \prod_{i=1}^{4} \frac{r_{i}}{\sigma_{i}^{2}} \exp \left(-\frac{1}{2|\Omega|} \sum_{i=1}^{4} \frac{r_{i}^{2}\left|\Phi_{i}\right|}{\sigma_{i}^{2}}\right) \int_{0}^{2 \pi} \int_{0}^{2 \pi} \exp \left[L_{13} \cos \left(t_{1}\right)+L_{14} \cos \left(t_{2}\right)+L_{34} \cos \left(t_{2}-t_{1}\right)\right] \\
& \quad \cdot I_{0}\left(\sqrt{L_{12}^{2}+L_{23}^{2}+L_{24}^{2}+2 L_{12} L_{23} \cos \left(t_{1}\right)+2 L_{12} L_{24} \cos \left(t_{2}\right)+2 L_{23} L_{24} \cos \left(t_{1}-t_{2}\right)}\right) d t_{1} d t_{2} .
\end{aligned}
$$

The submatrices involved are denoted as follows:

$$
\Phi_{i}=\left[\begin{array}{ccc}
1 & \rho_{j k} & \rho_{j l} \\
\rho_{j k} & 1 & \rho_{k l} \\
\rho_{j l} & \rho_{k l} & 1
\end{array}\right], \quad L_{i j}=\frac{r_{i} r_{j}}{\sigma_{i} \sigma_{j}}\left[\begin{array}{ccc}
\rho_{i j} & \rho_{j k} & \rho_{j l} \\
\rho_{i k} & 1 & \rho_{k l} \\
\rho_{j l} & \rho_{k l} & 1
\end{array}\right], \quad \Omega=\left[\begin{array}{cccc}
1 & \rho_{12} & \rho_{13} & \rho_{14} \\
\rho_{12} & 1 & \rho_{23} & \rho_{24} \\
\rho_{13} & \rho_{23} & 1 & \rho_{34} \\
\rho_{14} & \rho_{24} & \rho_{34} & 1
\end{array}\right] .
$$

The initial four-dimensional integral can once more be evaluated for the first two variables, after which numerical methods are necessary. We are interested in the impact of dimensionality on the accuracy of the proposed approximations and their computational effort. In this section, we will therefore assess the performance of both the integration and series expansion approaches for four-dimensional benchmark problems.

We begin with a test problem equivalent to the first example of the three-dimensional test section. By conveniently choosing the right parameters, we once more obtain a cofactor matrix for which a closed form of the density function does in fact exist. We have chosen $\sigma_{i}^{2}=0.8$ for $i=1, \ldots, 4$ and the covariances $\rho_{1,2}=-0.6123724, \rho_{1,3}=0.4082483, \rho_{1,4}=-0.2500000, \rho_{2,3}=-0.6666667, \rho_{2,4}=0.4082483$, $\rho_{3,4}=-0.6123724$. This results in the desired cofactor matrix in 26 .

$$
C=\left(\begin{array}{cccccccc}
0.08 & 0 & 0.04 & 0 & 0 & 0 & 0 & 0 \\
0 & 0.08 & 0 & 0.04 & 0 & 0 & 0 & 0 \\
0.04 & 0 & 0.08 & 0 & 0.04 & 0 & 0 & 0 \\
0 & 0.04 & 0 & 0.08 & 0 & 0.04 & 0 & 0 \\
0 & 0 & 0.04 & 0 & 0.08 & 0 & 0.04 & 0 \\
0 & 0 & 0 & 0.04 & 0 & 0.08 & 0 & 0.04 \\
0 & 0 & 0 & 0 & 0.04 & 0 & 0.08 & 0 \\
0 & 0 & 0 & 0 & 0 & 0.04 & 0 & 0.08
\end{array}\right) .
$$

The outcome of this experiment listed in Table 5 is highly reminiscent of the three-dimensional case. Once more the series expansion collapses to the true closed form, with the absolute errors somewhere in the order of magnitude of the machine precision. As we were expecting an outcome of the accuracy like this, 
we are using this predominantly as a benchmark to assess the computational effort of the two-dimensional integration techniques.

\begin{tabular}{|c|c|c|c|c|c|c|c|}
\hline $\begin{array}{l}f= \\
15\end{array}$ & TYPE & $\mathrm{AAE}$ & ARAE & AGAE & MAE & EVAL (s) & SETUP $(\mathrm{s})$ \\
\hline \multirow{3}{*}{ 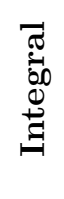 } & Romberg & $6.49500 \mathrm{E}-10$ & $2.20304 \mathrm{E}-05$ & $6.49500 \mathrm{E}-06$ & $3.72010 \mathrm{E}-08$ & 8401.97999 & 0.00000000 \\
\hline & Quadrature & 3.30873E-10 & $4.48268 \mathrm{E}-06$ & 3.30873E-06 & 7.44042E-09 & 19.2610001 & 0.00000000 \\
\hline & Cubature & $3.84581 \mathrm{E}-10$ & 1.15519E-05 & $3.84581 \mathrm{E}-06$ & $1.90177 \mathrm{E}-08$ & 176.161000 & 0.00000000 \\
\hline \multirow{8}{*}{ 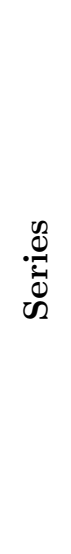 } & Closed & 7.46334E-19 & $9.11784 \mathrm{E}-15$ & 7.46334E-15 & $5.55112 \mathrm{E}-17$ & 0.19300008 & 0.00000000 \\
\hline & Series 3 & $0.00000 \mathrm{E}+00$ & $0.00000 \mathrm{E}+00$ & $0.00000 \mathrm{E}+00$ & $0.00000 \mathrm{E}+00$ & 5.84400010 & 0.77800012 \\
\hline & Series 6 & $0.00000 \mathrm{E}+00$ & $0.00000 \mathrm{E}+00$ & $0.00000 \mathrm{E}+00$ & $0.00000 \mathrm{E}+00$ & 22.7339999 & 7.96700001 \\
\hline & Series 9 & $0.00000 \mathrm{E}+00$ & $0.00000 \mathrm{E}+00$ & $0.00000 \mathrm{E}+00$ & $0.00000 \mathrm{E}+00$ & 60.3980000 & 49.8190000 \\
\hline & Series 12 & $0.00000 \mathrm{E}+00$ & $0.00000 \mathrm{E}+00$ & $0.00000 \mathrm{E}+00$ & $0.00000 \mathrm{E}+00$ & 126.354362 & 3.33145900 \\
\hline & Series 15 & $0.00000 \mathrm{E}+00$ & $0.00000 \mathrm{E}+00$ & $0.00000 \mathrm{E}+00$ & $0.00000 \mathrm{E}+00$ & 227.829000 & 9.79321837 \\
\hline & Series 18 & $0.00000 \mathrm{E}+00$ & $0.00000 \mathrm{E}+00$ & $0.00000 \mathrm{E}+00$ & $0.00000 \mathrm{E}+00$ & 377.296999 & 25.1501333 \\
\hline & Series 21 & $0.00000 \mathrm{E}+00$ & $0.00000 \mathrm{E}+00$ & $0.00000 \mathrm{E}+00$ & $0.00000 \mathrm{E}+00$ & 583.917999 & 55.3730833 \\
\hline
\end{tabular}

Table 5: Performance table for four-dimensional approximations.

As a second example we have chosen random covariance values, to allow for a more general case. The variances were chosen as $\sigma_{1}^{2}=0.5, \sigma_{2}^{2}=1, \sigma_{3}^{2}=1.5, \sigma_{4}^{2}=1$, and the correlations as $\rho_{1,2}=0.1, \rho_{1,3}=0.05$, $\rho_{1,4}=-0.1, \rho_{2,3}=0.025, \rho_{2,4}=0.2, \rho_{3,4}=-0.01$. Furthermore, we have reduced the grid size to $f=15$ in each dimension, leading to the evaluation of $15^{4}=50625$ nodes. As the additional dimension severely increased evaluation times of individual nodes, practical considerations and the fact that additional nodes did not change the error measures to any noteworthy degree led us to this decision. 


\begin{tabular}{|c|c|c|c|c|c|c|c|}
\hline $\begin{array}{l}f= \\
15\end{array}$ & TYPE & $\mathrm{AAE}$ & ARAE & AGAE & MAE & EVAL (s) & SETUP (s) \\
\hline \multirow{3}{*}{$\begin{array}{l}\widetilde{\sigma} \\
\stackrel{0}{0} \\
\stackrel{0}{0} \\
\stackrel{\Xi}{\Xi}\end{array}$} & Romberg & $2.57190 \mathrm{E}-10$ & $4.24838 \mathrm{E}-06$ & $2.57190 \mathrm{E}-06$ & 5.22977E-09 & 8260.27200 & 0.00000000 \\
\hline & Quadrature & $2.48131 \mathrm{E}-10$ & $3.24287 \mathrm{E}-06$ & $2.48131 \mathrm{E}-06$ & $5.21507 \mathrm{E}-09$ & 17.8032000 & 0.00000000 \\
\hline & Cubature & $3.33823 \mathrm{E}-10$ & $9.27479 \mathrm{E}-06$ & $3.33823 \mathrm{E}-06$ & $1.30708 \mathrm{E}-07$ & 108.509999 & 0.00000000 \\
\hline \multirow{8}{*}{ 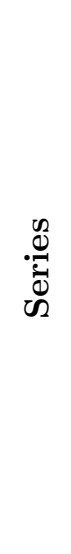 } & Series 1 & $4.87702 \mathrm{E}-05$ & $4.72348 \mathrm{E}+00$ & $4.87702 \mathrm{E}-01$ & 8.93641E-04 & 0.23400021 & 0.00000000 \\
\hline & Series 3 & $1.34680 \mathrm{E}-07$ & 8.89451E-02 & $1.34680 \mathrm{E}-03$ & 2.91429E-06 & 5.74760008 & 0.76440001 \\
\hline & Series 6 & $3.11428 \mathrm{E}-10$ & 8.63636E-04 & $3.11428 \mathrm{E}-06$ & 9.64595E-09 & 22.3430001 & 7.84459996 \\
\hline & Series 9 & $5.77737 \mathrm{E}-13$ & $4.62287 \mathrm{E}-06$ & $5.77737 \mathrm{E}-09$ & $3.25826 \mathrm{E}-11$ & 59.1546001 & 48.8952000 \\
\hline & Series 12 & $8.40614 \mathrm{E}-16$ & $1.47151 \mathrm{E}-08$ & $8.40614 \mathrm{E}-12$ & $9.05531 \mathrm{E}-14$ & 124.848599 & 196.241199 \\
\hline & Series 15 & $9.48983 \mathrm{E}-19$ & $2.96553 \mathrm{E}-11$ & $9.48983 \mathrm{E}-15$ & $1.64584 \mathrm{E}-16$ & 224.137799 & 582.901400 \\
\hline & Series 18 & $8.47948 \mathrm{E}-22$ & $4.02875 \mathrm{E}-14$ & 8.47948E-18 & 2.32934E-19 & 370.081800 & 1469.78179 \\
\hline & Series 21 & $4.40060 \mathrm{E}-25$ & $3.51555 \mathrm{E}-17$ & $4.40060 \mathrm{E}-21$ & $1.69407 \mathrm{E}-21$ & 570.203400 & 3267.89159 \\
\hline
\end{tabular}

Table 6: Performance table for four-dimensional approximations.

The results in Table 6 are more revealing about the approximations' behaviours in higher dimensions. While the integration approach maintains roughly the same accuracy as in the constructed experiment, the series approach matches and surpasses the results with only 6 sum terms. This marks a considerable further intensification of the disparity of both methods performance we had observed in the three-dimensional case. Due to the numerical integration methods being increasingly computationally expensive in higher dimensions, the rift between the performances of both methods grows even further.

The recorded computation times appear to confirm these observations. The evaluation of the series expansion takes only a fraction of the evaluation time the numerical integration requires. The setup time, while having been recorded for consistency, may once more be neglected. As we earlier elaborated, the necessary coefficients in the setup process have to be computed only once, regardless of covariance matrix. In the following discussion we refer to the Romberg method only, as the implementations of the cubature methods are once more merely wrappers for other, faster coding languages and can therefore not be directly compared to a pure $\mathrm{R}$ implementation. However, we do acknowledge a potential practical use, while keeping in mind a $\mathrm{C}$ or Matlab implementation of the series representation would likely surpass the integration approach in terms of accuracy and efficiency. At 9 sum terms we have surpassed the only integration method implemented in $\mathrm{R}$, with an average error of $5.77 \times 10^{-13}$ versus $2.57 \times 10^{-10}$. Remarkably, the computation time stands at $59.15 \mathrm{~s}$ against the integration methods' $8260.27 \mathrm{~s}$, reducing the computational time by 140 times. We observe that at 6 sum terms and an average error of $\sim 3.1 \times 10^{-10}$ we are in the same accuracy range of the integration approaches at $\sim 2.5-3.3 \times 10^{-10}$, while approaching the evaluation time of the $\mathrm{C}$ code based quadrature evaluation at $\sim 18 s$ with $\sim 22.3 s$ for the series expansion.

As for the 3-dimensional examples, we test another example covariance matrix with high correlation values in four dimensions, to assess the influence on the accuracy of the approximations. The variances are chosen as $\sigma_{1}=0.5, \sigma_{2}=1, \sigma_{3}=1.5$ and $\sigma_{4}=1$. The correlations are $\rho_{1}=0.7, \rho_{2}=0.8, \rho_{3}=0.9, \rho_{4}=0.7$, $\rho_{5}=0.8$ and $\rho_{6}=0.7$. 
When reviewing the results in Table 7, we have once more omitted the Romberg integration method due to slow convergence/numerical instability (the method failed to compute the grid points within 24h). The comparison here is therefore somewhat problematic, as the quadrature and cubature methods rely on MatLab and $\mathrm{C}$ code subroutines as stated earlier. We therefore lack a direct comparison between integration and series expansion methods within the same implementation environment.

\begin{tabular}{|c|c|c|c|c|c|c|c|}
\hline $\begin{array}{l}f= \\
15\end{array}$ & TYPE & $\mathrm{AAE}$ & ARAE & AGAE & MAE & EVAL (s) & SETUP (s) \\
\hline \multirow{3}{*}{ 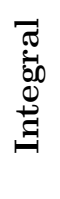 } & Romberg & - & - & - & - & NA & NA \\
\hline & Quadrature & $1.17185 \mathrm{E}-09$ & $1.67902 \mathrm{E}-05$ & $5.93247 \mathrm{E}-05$ & $3.15848 \mathrm{E}-08$ & 32.5610001 & 0.0000000 \\
\hline & Cubature & $3.97861 \mathrm{E}-09$ & $1.01483 \mathrm{E}-04$ & $2.01417 \mathrm{E}-04$ & $7.60076 \mathrm{E}-05$ & 1144.98699 & 0.0000000 \\
\hline \multirow{8}{*}{ 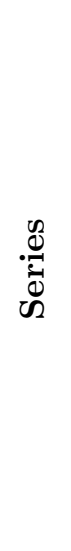 } & Series 1 & $3.87385 \mathrm{E}-03$ & $5.34388 \mathrm{E}+01$ & $1.96113 \mathrm{E}+02$ & $1.33733 \mathrm{E}-01$ & 0.73600016 & 0.0000000 \\
\hline & Series 3 & $9.62893 \mathrm{E}-04$ & $1.67841 \mathrm{E}+01$ & $4.87465 \mathrm{E}+01$ & 3.63151E-02 & 58.2030001 & 1.7140000 \\
\hline & Series 6 & $1.68243 \mathrm{E}-04$ & $3.79967 \mathrm{E}+00$ & $8.51730 \mathrm{E}+00$ & 7.92697E-03 & 184.078000 & 22.886999 \\
\hline & Series 9 & $2.39765 \mathrm{E}-05$ & $6.97499 \mathrm{E}-01$ & $1.21381 \mathrm{E}+00$ & $1.56577 \mathrm{E}-03$ & 481.630999 & 109.68700 \\
\hline & Series 12 & 2.92533E-06 & $1.09059 \mathrm{E}-01$ & $1.48095 \mathrm{E}-01$ & $2.84394 \mathrm{E}-04$ & 1026.01399 & 472.93000 \\
\hline & Series 15 & $3.11890 \mathrm{E}-07$ & $1.48686 \mathrm{E}-02$ & $1.57894 \mathrm{E}-02$ & $4.79140 \mathrm{E}-05$ & 1793.01699 & 1425.0100 \\
\hline & Series 18 & $2.93815 \mathrm{E}-08$ & 1.78987E-03 & $1.48744 \mathrm{E}-03$ & 6.79694E-06 & 2527.42678 & 3748.9999 \\
\hline & Series 21 & $2.46319 \mathrm{E}-09$ & $1.91531 \mathrm{E}-04$ & $1.24699 \mathrm{E}-04$ & $9.21801 \mathrm{E}-07$ & 2958.49176 & 5274.3876 \\
\hline
\end{tabular}

Table 7: Performance table for four-dimensional approximations with high correlation covariance matrix.

We do notice however that the higher correlation values negatively influence the performance of all methods, both integration and series expansion based. Both quadrature and cubature integration methods lose some of their previous accuracy, increasing the AAE from $\sim 3 \times 10^{-10}$ to $1.17 \times 10^{-9} / 4 \times 10^{-9}$. Meanwhile the computation time has increased for the quadrature method by $100 \%$, whereas the cubature approach has increased tenfold.

The series approach converges significantly slower as well, as the errors seemingly decreased by $\sim 10^{-3}$ for an additional 3 sum terms. This has now been decreased to roughly $\sim 10^{-1}$ per 3 sum terms. We note that the computation time is still below the Romberg method's computation time for the lower correlation covariance matrix example. For the high correlation the Romberg method was not able to complete the computation within what we felt was a reasonable amount of time $(<24 h)$. We note once more that the quadrature and cubature methods are implemented in $\mathrm{C}$ and MatLab code, with only an $\mathrm{R}$ front end. This means the algorithms efficiency cannot be directly compared. Moving forward we will therefore implement the fairly straightforward algorithm of the series approximation in $\mathrm{C}$ code or a comparable language, as $\mathrm{R}$ itself is not suitable for practical purposes.

The performance behaviour and influence of sum terms become evident in Figure 2, where we see the accuracy of the series representation quickly surpassing the integration approaches, conserving a convergence in the order of $\sim 10^{-n}$, while preserving computational effort. 


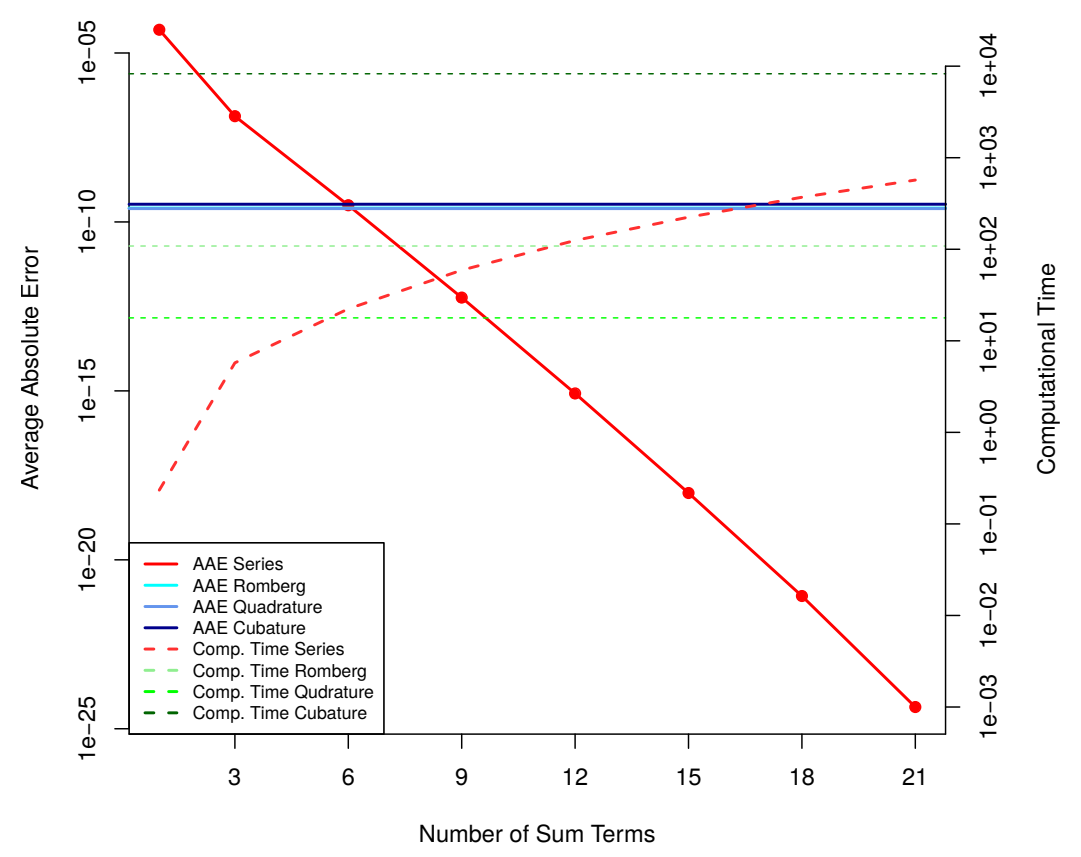

Figure 2: A graphical comparison between accuracy and runtimes of all tested four-dimensional approximation methods.

Paired with the more general applicability of the series presentation, its availability for arbitrary dimensions and with no restrictions on the composition of the covariance matrix (except for invertibility) the performance for three and four-dimensional cases heavily favoured the series representation. While these examples showcased the performance of the series expansion, we are predicting that this difference in performance may become more pronounced as more channels are added. However, due to the lack of comparable algorithms in higher dimensions, we are currently unable to investigate this suspicion further. We therefore conclude that the approach introduced in this paper is superior, and has the potential to enable new and yet untapped fields of application and research.

\section{Applications}

One of the most common applications for the Rayleigh distribution is the computation of outage probabilities. Generally this is done via the formula given in 28, which is the outage probability for a threedimensional setup. We are utilising the definition noted by Chen and Tellambura [4]:

$$
\begin{aligned}
P_{\text {out }}\left(\gamma_{t h}\right) & =\int_{0}^{\sqrt{\frac{\gamma_{t h} \Sigma_{1,1}}{\bar{\gamma}_{1}}}} \int_{0}^{\sqrt{\frac{\gamma_{t h} \Sigma_{2,2}}{\bar{\gamma}_{2}}}} \int_{0}^{\sqrt{\frac{\gamma_{t h} \Sigma_{3,3}}{\bar{\gamma}_{3}}}} f_{R}\left(r_{1}, r_{2}, r_{3}\right) d r_{1} d r_{2} d r_{3} \\
& =F_{R}\left(\gamma_{t h}, \gamma_{t h}, \gamma_{t h}\right),
\end{aligned}
$$

where $\gamma_{t h}$ denotes the threshold signal to noise ratio (SNR). We demonstrate how the series representations can be used to compute the probabilities in relation to the inverse average SNR ratio $\bar{\gamma}_{i}$ of a single channel. 

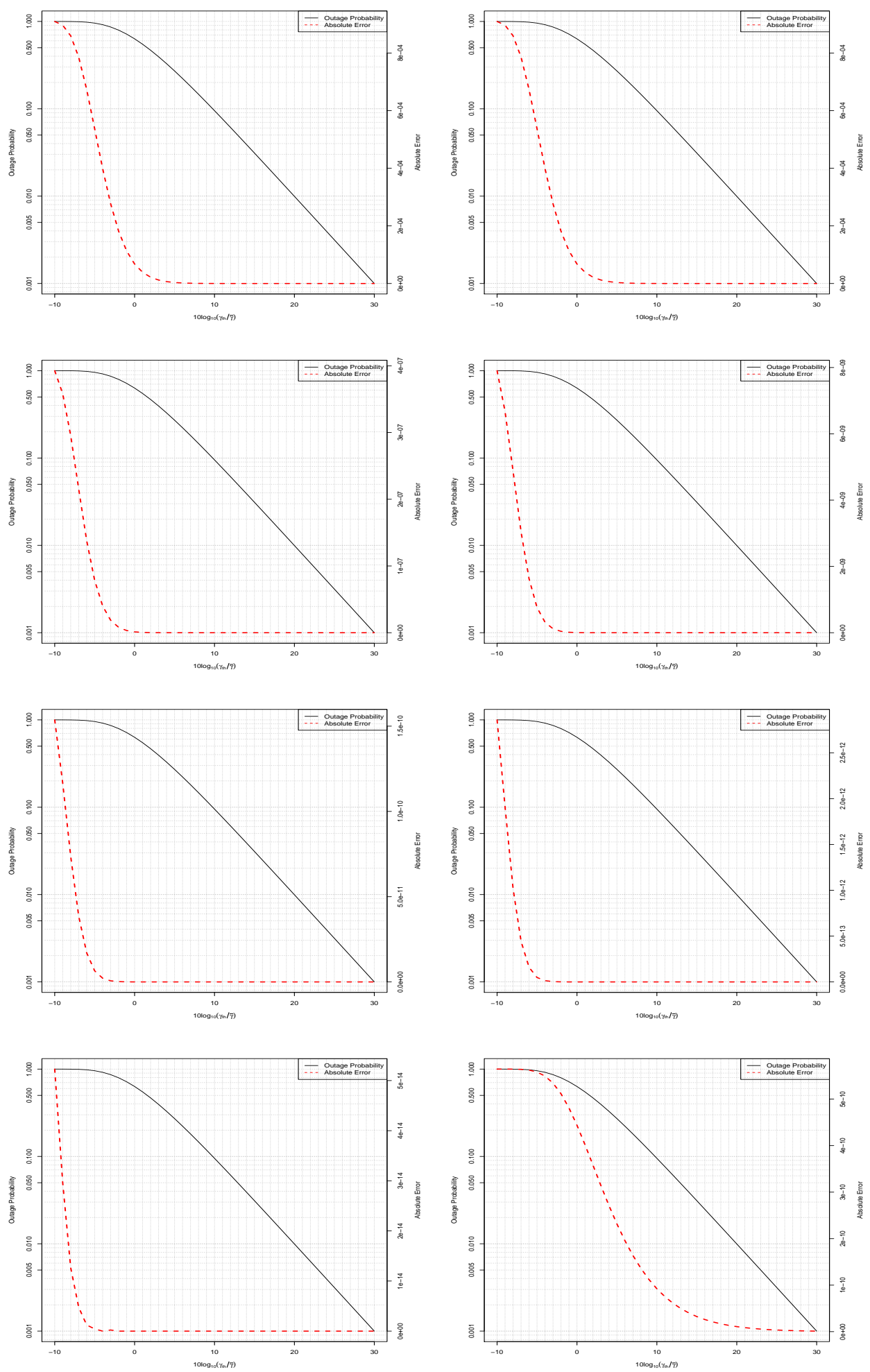

Figure 3: Outage probabilities versus average SNR with error distribution for all three-dimensional approximations: series expansion 3 terms (first row, left); series expansion 6 terms (first row, right); series expansion 9 terms (second row, left); series expansion 12 terms (second right, right); series expansion 15 terms (third row, left); series expansion 18 terms (third row, right); series expansion 21 terms (fourth row, left); integral based approximation (fourth row, right). 
In Figure 3 we see the results for each approximation approach. The structure and setup of the outage probability graphs have been taken from Simon and Alouini [13], and depict the SNR and the corresponding outage probability. The outage probability is in turn superimposed with the absolute error distribution across the SNR values, not only highlighting the overall quality of the approximation, but also the performance in relation to the input values. The overall shape of the error function remains similar for each approach, but we do notice that the series expansion of higher order performs significantly better than the integration approach. The slope of the error distribution changes drastically, and for $p=15$ sum terms, the absolute error approaches zero almost instantly. Unsurprisingly the performance results are largely in agreement with the observations made in the previous section, as only a layer of numerical integration has been added in this application.

\section{Conclusions}

In this paper, we have generalised the results of [1] to retrieve a series expansion for the Rayleigh distribution for arbitrary dimension, with no restrictions on the covariance matrix values. Additionally we have investigated the approximation's performance in simulation studies of three and four dimensions with a recent integration based method. The series expansion compared favourably in both accuracy and computational effort to the proposed benchmark approach. In higher dimensions the difference in performance became more pronounced as the numerical integration methods required added computational effort. The implementation of the series expansions and the integration methods are available in the $\mathrm{R}$ package DRAYL on CRAN [14].

In the previous section, we gave examples for applications in signal processing, by computing the outage probability of a three branch system through both [2]'s method and the series expansion, which matched our observations made during the performance analysis. This showed that the series approach remains stable and accurate for practical applications as well. In a next step we will extend the series expansion to include the cumulative distribution function (CDF) of Rayleigh distributions as well, eliminating the need for numerical integration. Given the frequent use of the CDF to compute outage probabilities, it would be desirable to have a more direct method of computation, instead of the numerical integration of density approximations. Additionally, a simpler version of the presented approximation may be available, as uncountable series expansions exist which may also be investigated, and provide a more accessible formula. This will also be part of upcoming projects, along with the development of a more practically suitable implementation of the existing algorithms, as our simple prototype in $\mathrm{R}$ leaves a lot of room for improvement and other languages and implementation techniques will be considered.

\section{Acknowledgments}

The authors would like to thank the Editor and the four referees for careful reading and comments which greatly improved the paper.

\section{References}

[1] M. Wiegand, S. Nadarajah; "A series representation for multidimensional Rayleigh distributions" International Journal of Communications Systems, Vol. 31, Issue 6, 2018

[2] N. C. Beaulieu, Y. Zhang; "New simplest exact forms for the 3-D and 4-D multivariate Rayleigh PDFs with applications to antenna array geometries" IEEE Transactions on Communications, Vol. 65, No. 9, 2017 
[3] C. Beard, M. Tekinay; "A method to construct infinite series representation of quadrivariate Rayleigh distribution" Preprint, April 2017

[4] Y. Chen, C. Tellambura; "Infinite series representations of the trivariate and quadrivariate Rayleigh distribution and their applications" IEEE Transactions on Communications, Vol. 53, 2005

[5] P. Beckmann; "Rayleigh distributions and its generalisations" Radio Science Journal of Research, Vol. 68D, No. 9, 1964

[6] C. Beaulieu, K. Hemachandra; "Novel simple forms for multivariate Rayleigh and Rician distributions with generalised correlation" IEEE Globecom, 2010

[7] K. N. Le; "Comments on "Distribution functions of selection combiner output in equally correlated Rayleigh, Rician, and Nakagami-m fading channels"” IEEE Transactions on Communications, Vol. 63, No. 12, pp. 5283-5287, 2015

[8] K. N. Le; "Distributions of multiuser-MIMO under correlated generalised-Rayleigh fading" Signal Processing, Vol. 150, No. 9, pp. 228-232, 2018

[9] K. N. Le; "Selection combiner output distributions of multivariate equally-correlated generalisedRician fading for any degrees of freedom" IEEE Transactions on Vehicular Technology, Vol. 67, No. 3, pp. 2761-2765, 2018

[10] K. N. Le; "On selection combiner output quadrivariate cumulative distribution functions in correlated Nakagami-m and Rayleigh fading" IEEE Communications Letters, Vol. 20, No. 9, pp. 17171720,2016

[11] S. O. Rice; "Mathematical analysis of random noise" The Bell System Technical Journal, Vol. 23, pp. 282-332, 1944

[12] K. S. Miller; “Complex Gaussian processes” SIAM Review, Vol. 11, pp. 544-567, 1969

[13] M. K. Simon, M. -S. Alouini; "A unified performance analysis of digital communication with dual selective combining diversity over correlated Rayleigh and Nakagami-m fading channels" IEEE Transactions on Communications, Vol. 47, No. 1, January 1999

[14] M. Wiegand; "DRAYL: Approximations for multivariate Rayleigh density functions" Comprehensive R Archive Network (CRAN) 